\title{
A NEW PROSTHESIS FOR DISARTICULATION AT THE HIP
}

\author{
LeON Gillis, ${ }^{*}$ London, EnGland
}

From Queen Mary's Hospital, Roehampton, London

The locomotor problem in bilateral disarticulation at the hip is serious because there is no natural motive power left to help swing either limb forward, and the physiological mechanism used in unilateral disarticulation is no longer available. The only motive power is pelvic and spinal in origin. To overcome this, a new "swing door" hinge prosthesis has been developed, which is particularly suitable for bilateral disarticulation, but also has advantages in the case of unilateral disarticulation.

It acts in the following manner. The weight of the artificial limb is held in balanced spring-loaded tension so that when a step is taken with one limb, part of the body weight is transferred anterior to the line of weight transmission in the other leg, forcing the top of the hinge open and giving a natural lengthening to the weight-bearing limb. When the weight has been taken off the limb, in the swing phase, the top hinge closes and the lower spring, which is now in tension, swings the limb forward naturally. Mechanical limiters. which incorporate a lock to allow for the sitting position, control the length of the forward movement and give a positive stop when the patient is standing.

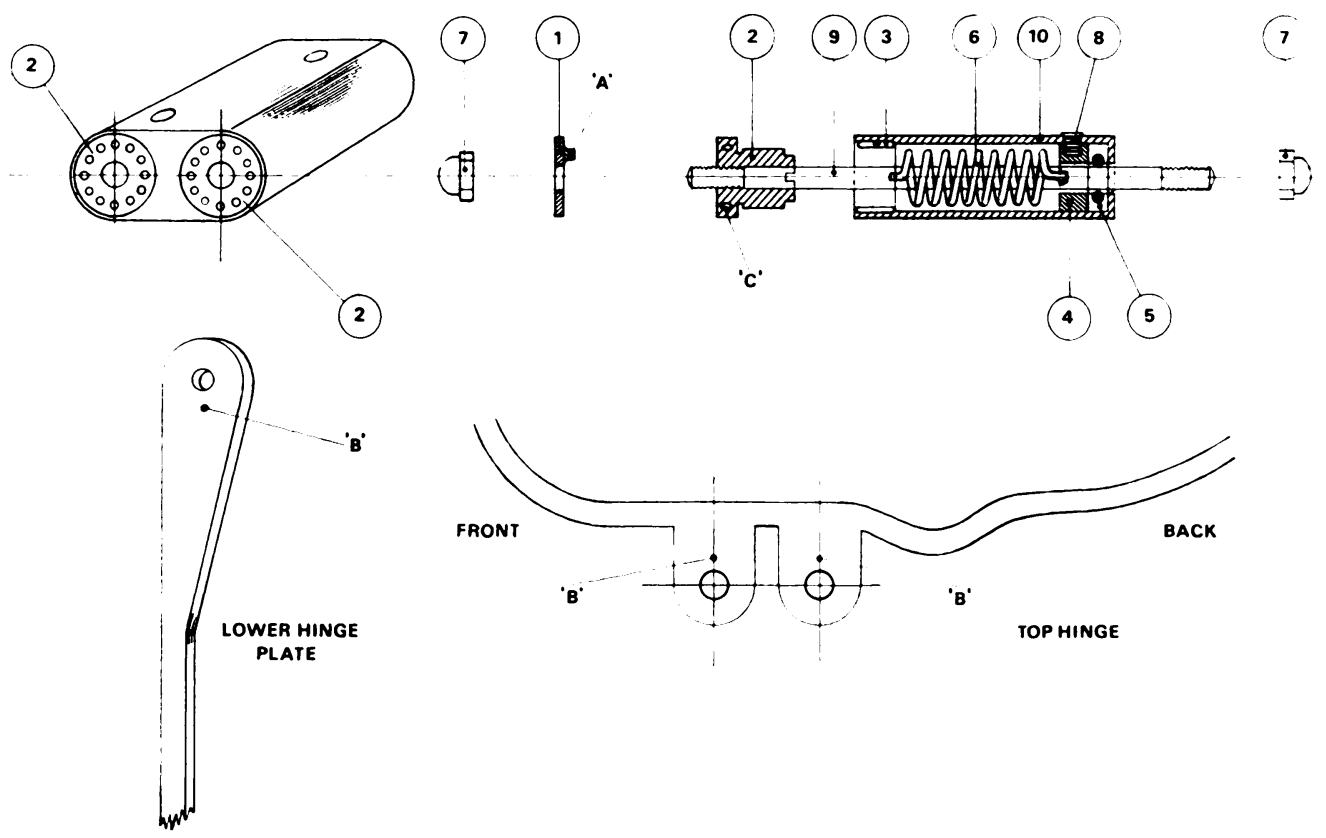

Fig. 1

The double-action power hinge has a main body (10) with two parallel bores machined to house the needle bearings (3), coil springs (6), static spring retainer bushes (4), and ball races (5). They are machined on 28 millimetres centres allowing a small clearance between the top and lower hinge plates, which are made of high-tensile steel. The top hinge plate is riveted to the socket with a clearance to allow a rubber buffer to be inserted. The lower hinge plate is made to suit the standard types of artificial limbs.

The loading of the hinges is as follows. The spring retained bush (2), which rotates in the needle bearings, is turned by means of a tommy bar in the holes provided (C) against the pressure of the spring. It is then locked in tension by a locking plate (1). The pin (A) on the locking plate passes through hole (B) in the hinge plate and into one of the holes in the retainer bush (2). The hinge assembly is held in position by the spindle (9) and retained by shake-proof nuts (7).

* Deceased August 9, 1967 


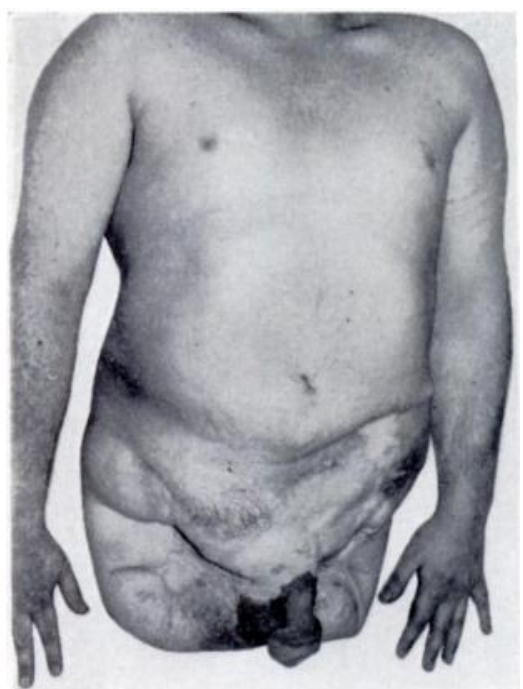

FiG. 2

FIGS. 2 AND 3

Figure 2-The patient's trunk. Figure 3-The patient standing.

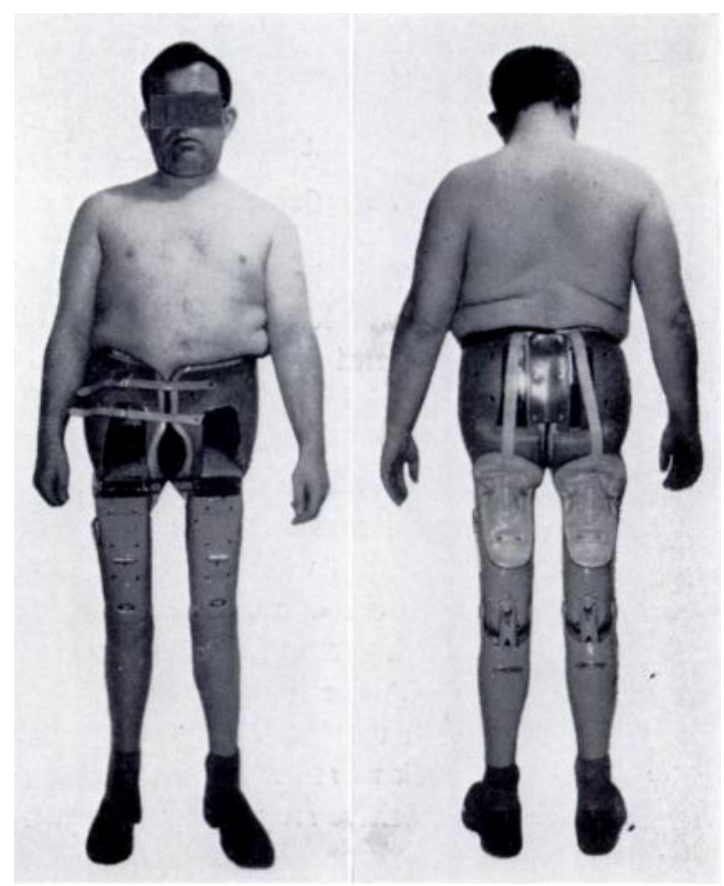

FIG. 3

The joint consists of two torsion springs, one a right hand and the other a left hand spring, flexing and extending the joint respectively, and balancing each other and the weight of the limb at a neutral angle. The housings of each spring are hinged to a flap which maintains them in a spaced relationship, the housing of one spring being secured to the bucket and that of the other spring to the artificial limb. The longitudinal axis of each spring lies in a plane transverse to the longitudinal axis of the limb, with one spring situated in front of the other. The housing of each spring comprises a hinge leaf coupled to the spring and arranged so that relative movement between the plate and one leaf causes stressing of the corresponding spring. The springs are maintained under a slight torque to hold the leaves together above and below the spacing plate. The rear leaf is secured to the bucket and the forward leaf to the artificial limb (Fig. 1).

If the prosthesis is used in the case of single disarticulation at the hip, its action is as follows. When the normal leg is moved forward, the artificial leg moves backwards, and as the rear spring leaf is attached to the hip socket the spacing plate and front spring rotate around the rear spring axis, tightening the rear spring. So when the weight is taken off the artificial limb the loaded spring asserts itself, causing forward movement of the artificial limb until this reaches the position of equilibrium in a straight line with the socket. The weight and impetus of the body moving forward then cause the limb to swing further forwards and the forward leaf to rotate about the axis of the forward spring, continuously tightening it as the weight is placed upon the limb until such time as the natural limb is once more moved forward, when, as the weight is taken off the artificial limb, the forward spring reasserts itself and moves the limb back to its equilibrium position, after which the whole cycle is repeated. It has been found that some mechanical assistance during extension at the hip still further assists the amputee, and a hinge incorporating a coil spring has been designed to do this.

As this prosthesis produces flexion of the hip from the fully extended position without the aid of the opposite hip extension, it is of special value in a case of bilateral disarticulation. 


\section{CASE REPORT}

A man of twenty-six slipped between two carriages of a train and sustained multiple injuries including 1) amputation of both legs, two or three inches of each femur remaining; 2) loss of lower abdominal skin; 3 ) loss of testes and scrotum and loss of skin of the penis. After resuscitation, extensive wound cleansing, ligation of the femoral vessels and removal of the femoral stumps to obtain primary skin closure, what loose skin remained was sutured to the abdominal wall. Three weeks later further wound cleansing and removal of sloughs was carried out and after a further three weeks the raw areas remaining were successfully grafted with split skin taken from the arm (Fig. 2).

The patient has been fitted with the double hinge appliance described above. $\mathrm{He}$ is now personally independent in the application of the prosthesis, dressing and bathing. $\mathrm{He}$ has a good standing balance over limited periods of thirty minutes (Fig. 3). He can walk a quarter of a mile unaided with elbow crutches and can climb stairs if there are hand rails on both sides of the staircase. Providing the arms of a chair are at the correct height he can get up from a chair without difficulty. These activities are considerably in advance of those achieved with the older appliances.

\section{DISCUSSION}

The conventional artificial limb for unilateral disarticulation was formerly a tilting table. There were drawbacks to this type of prosthesis. Firstly, the socket was attached to the torso by a narrow pelvic band or bands, and this method of suspension was insecure, often allowing the socket to twist. Secondly, the artificial hip was a spindle joint and was placed between the socket and the thigh piece, horizontal and inferior to the socket. This made sitting lop-sided. Thirdly, the joint was locked during walking. Swinging of the artificial limb was achieved by the spinal muscles tilting the pelvis while enabling the other hip to be hyperextended. The gait was unphysiological and ungainly.

The Canadian prosthesis was introduced to overcome some of these objections. This consists of a socket fitting the torso with a broad extension embracing the other side of the body. A spindle joint is placed between the socket and the thigh piece, which allows a small degree of flexion and extension of the hip during walking because it is not locked. It also permits a good sitting position.

The double hinge prosthesis-The aim of the new appliance is to replace the work of the spinal muscles with springs controlling the swinging of the limb at the hip and so produce a heavy powered mechanism giving a natural gait once the inertia of the apparatus is overcome. It is essential that the socket is securely attached to the body. In taking casts for these moulded leather sockets, care must be taken to shape the plaster cast properly, not only in front and behind the ischial tuberosity, but over the iliac crests. This enables the ischial tuberosity and the gluteal muscles to obtain a better grip on the socket. This method of attachment is a distinct improvement, because the socket fits snugly in both unilateral and bilateral amputations and feels a part of the patient's own body.

\section{SUMMARY}

1. A prosthesis which is useful in unilateral disarticulations of the hip and highly advantageous in cases of bilateral disarticulation is described.

2. It makes use of the principle of a swing door and makes walking possible with the assistance of elbow crutches in cases of bilateral hip disarticulation and amelia.

My thanks are due to the late Colonel E. S. Wheatley and Mr G. R. Parry of the Limb Fitting Centre, Queen Mary's Hospital, Roehampton, for their co-operation in this work. The appliance was designed by the author and constructed by Messrs J. E. Hanger \& Co. Ltd. whose co-operation throughout has been much appreciated, particularly that of the Managing Directors, Mr J. B. Waggott and Mr H. B. Wells. I would also like to thank $\mathrm{Mr} \mathrm{J}$. G. Bonnin who has helped me in rewriting this article.

VOL. $50 \mathrm{~B}$, NO. 2, MAY 1968 